\title{
The inflammatory cytokine IL-22 promotes murine gliomas via proliferation
}

\author{
XIGUO LIU, JUNJING YANG and WANKAI DENG \\ Department of Head and Neck and Neurosurgery, Hubei Cancer Hospital, Wuhan, Hubei 430079, P.R. China
}

Received July 30, 2015; Accepted May 16, 2016

DOI: $10.3892 /$ etm.2017.4059

\begin{abstract}
Interleukin (IL)-22 is newly identified proinflammatory cytokine involved in the T helper (Th)17 and Th22 response. However, the possible role of IL-22 in glioma remains uncertain. The results of the present study demonstrated higher expression levels of IL-22 and the receptor IL-22BP in the brain of GL261 glioma-inoculation mice, suggesting the regulatory role of IL-22 in glioma. Injection of IL-22 increased the severity of glioma in vivo and higher expression levels of IL-6, IL-1 $\beta$ and tumor necrosis factor (TNF)- $\alpha$ were detected in the brain using ELISA following IL-22 injection. To elucidate the mechanism underlying the effects of IL-22, the present study aimed firstly to determine the expression levels of IL-22 receptor in a glioma cell line via reverse transcription quantitative polymerase chain reaction. IL-22 treatment significantly increased the expression levels of signal transducer and activator of transcription (STAT)3 and the mRNA expression levels of STAT6 compared with the vehicle control. These results suggested that IL-22 may activate the Janus kinase (JAK)/STAT signaling pathway in glioma. Furthermore, IL-22 positively regulated the proliferation of glioma, consistent with its role in vivo. Conversely, IL-22-deficient mice exhibited prolonged survival compared with wild-type (WT) mice, and the expression levels of inflammatory cytokines were decreased in the brain of IL-22 knock-out (KO) mice compared with WT mice. Concordant with these results, it was observed that IL-22-neutralising antibody was able to increase the survival of mice with glioma and attenuate the disease by significantly reducing the cytokine levels in the brain. In conclusion, the results of the present study demonstrated that expression levels of IL-22 in the brain of mice with glioma may enhance symptoms due to the increased cytokine production of IL- 6 , IL- $1 \beta$ and TNF- $\alpha$; this is consistent with IL-6/JAK/STAT signalling activation in vitro. Decreasing the expression levels of IL-22, achieved
\end{abstract}

Correspondence to: Dr Xiguo Liu, Department of Head and Neck and Neurosurgery, Hubei Cancer Hospital, 116 Zhuodaoquan South Road, Wuhan, Hubei 430079, P.R. China

E-mail: liuxiguo201501@126.com

Key words: interleukin-22, gliomas, proliferation either with IL-22-KO mice or IL-22-neutralising antibody demonstrated protective effects on glioma development. Therefore, IL-22 may serve as a potential therapeutic target for glioma.

\section{Introduction}

Malignant gliomas are brain tumors characterized by high proliferation and escape from immunosurveillance via numerous mechanisms. Clinical vaccination trials aimed to decrease immune tolerance against high grade gliomas have been conducted $(1,2)$. Tumor regression appears to be associated with the absence of a large tumor mass secreting tumor growth factor (TGF)- $\beta 2$ and on the maturation status of dendritic cells inside and around the tumor $(3,4)$. Therapeutic strategies targeting the immune response in the brain are therefore of particular interest in the search for efficient treatments of malignant gliomas. The central nervous system (CNS) is considered to be a unique immunological site due to the presence of the blood-brain barrier, and low immune reactivity prevents accidental inflammation within the CNS (5-7). However, in the case of a CNS tumor, strong immune responses against the invading pathogens develop indicating that potent immune responses may occur against tumor homeostasis (8).

Interleukin (IL)-22 is a major cytokine member of the IL-10 cytokine super family, which also includes IL-19, IL-22, IL-22, IL-24, IL-26, IL-28 and IL-29, and is secreted by $\mathrm{T}$ helper $(\mathrm{Th}) 17(9,10)$. However, IL-22 exhibits potent pro-inflammatory properties, unlike IL-10 (11). A previous study reported that IL-22 induced by IL-23 had an important role in psoriasis, since IL-22 was demonstrated to be required for imiquimod-induced psoriasiform skin inflammation in mice (11). IL-22 triggers an inflammatory response by activating signal transducer and activator of transcription (STAT)3 signaling, and is able to promote hepatocellular carcinoma (HCC) tumor-infiltrated leukocytes due to high expression in this cell type (12). However, the effect of IL-22 on brain tumors remains to be elucidated. In 2002, a study reported that IL-22 is able to positively regulate signaling pathways such as p38/extracellular signal-regulated kinase/c-Jun N-terminal kinase/mitogen-activated protein kinase and Janus kinase (JAK)/STAT in hepatoma cells (13). However, few papers report its role in brain tumors. IL-22 was observed to have an anti-apoptosis effect in lung cancer, acting in an autocrine manner (14). In addition, IL-22 was demonstrated to trigger 
inflammation and drive tumor progression via IL-22R1 signaling in large cell lymphoma (15). In HCC, long term STAT3 activation by IL-22 may promote tumor growth by targeting damaged hepatocytes and tumor cells, similar to HCC promotion by IL-6 (12). However, self-reactive Th cells coexpress IL-17 and IL-22, and the latter does not appear to be directly involved in autoimmune pathogeneses of the CNS $(16,17)$.

\section{Materials and methods}

Cell culture and drug treatment. The GL261 murine glioma cell line was obtained from the American Type Culture Collection (Manassas, VA, USA). Cells were cultured in vitro at $37^{\circ} \mathrm{C}(5 \%$ $\mathrm{CO}_{2}$ ) in Iscove's Modified Dulbecco's Medium (Invitrogen; Thermo Fisher Scientific, Inc., Waltham, MA, USA) supplemented with $10 \%$ fetal calf serum (Sigma-Aldrich; Merck Millipore, Darmstadt, Germany), $1 \% 100 \mathrm{U} / \mathrm{ml}$ penicillin and $1 \% 100 \mathrm{~g} / \mathrm{ml}$ streptomycin (Invitrogen; Thermo Fisher Scientific, Inc.) and $20 \mathrm{M} \beta$-mercaptoethanol (complete medium). IL-22 protein was purchased from PeproTech, Inc. (Rocky Hill, NJ, USA). Anti-IL-22 neutralising polyclonal rabbit antibodies (ab109819) were purchased from Abcam (Cambridge, MA, USA).

Animal model. A total of 50 female C57BL/6 mice (age, 6-12 weeks; weight, 20-25 g) were obtained from Charles River Laboratories (Wilmington, MA, USA). A brain tumor model was set up as described previously (11). A total of 1x10 4 GL261 glioma cells were washed twice in phosphate-buffered saline (PBS) and adjusted to $5 \mu \mathrm{lPBS}$ in a 26-gauge Hamilton syringe. The mice were anesthetized with $1.2 \%$ isoflurane (792632; Sigma-Aldrich). Following shaving, an incision was made in the scalp, and a burr hole was made in the skull $2 \mathrm{~mm}$ lateral to the midline and $2 \mathrm{~mm}$ anterior to the bregma using a dental drill. Subsequently, GL261 glioma cells were incubated with anti-IL-22 neutralising polyclonal rabbit antibodies at $4^{\circ} \mathrm{C}$ for $24 \mathrm{~h}$. Following neutralization, GL261 glioma cells were injected over $1 \mathrm{~min}$ at a depth of $2.5 \mathrm{~mm}$ below the dura mater into the right cerebral hemisphere. The mice were observed daily and sacrificed by cervical dislocation when characteristic symptoms such as hunched posture, reduced mobility, and significant weight loss $(20 \%)$ occurred within 10 days of glioma implantation. Animals without such symptoms were regarded as long-term survivors after 90 days. A total of 50 IL-22-deficient [knock-out (KO)] mice were generated by targeting exons 1-3 and backcrossed onto C57BL/6 $>8$ times, as described previously (16). The targeting vector was constructed to replace the exons $1 \mathrm{a}, 1 \mathrm{~b}$, 2 , and a part of exon 3 of the IL-22a gene by a neomycin-resistant gene. A 5' arm of 1,521 bp was amplified using a mutated sense primer with a XhoI site (5'-CTTCGGCTCGAGATGGCCAC-3') and a mutated antisense primer also containing a XhoI site (5'-GCCCTCGAGACACCAGGGTT-3') to allow the direct insertion into the pPNT vector. The $3^{\prime}$ arm consisted of a 3,559-bp KpnI fragment, containing the end of exon 3 and exon 4 , and was cloned.

Mice were divided into GL261 glioma implantation + IL-22 and GL261 glioma implantation + vehicle groups ( $n=6$ per group) and the brain tissues were harvested. The mice were bred under specific pathogen-free conditions, and all experimental protocols were approved by the Institutional
Animal Care and Use Committee of Hubei Cancer Hospital (Wuhan, China).

Evaluation of proliferation. GL261 glioma cells were analyzed for proliferation using a Cell Counting kit-8 (CCK8; Dojindo Molecular Technologies, Inc., Shanghai, China). Cells were seeded into 96 -well plates at densities of $1 \times 10^{4}$ cells/well, and incubated in a humidified atmosphere containing $5 \% \mathrm{CO}_{2}$ and 95\% air overnight. Normal cell medium containing either IL-22 or $0.01 \mathrm{M}$ PBS vehicle at the desired concentration were added to the cells. After $72 \mathrm{~h}$ incubation, $10 \mu \mathrm{l}$ WST- 8 from CCK8 (5 g/l in PBS) was added. The plates were incubated for $4 \mathrm{~h}$ and the blue dye formed was dissolved in $100 \mu \mathrm{l}$ dimethyl sulfoxide. Absorbance at $450 \mathrm{~nm}$ was recorded using an ELISA reader.

Evaluation of cell death. The cells were stained with propidium iodide (PI; BD Biosciences, San Jose, CA, USA) and cell death was evaluated according to the manufacturer's instructions. Briefly, cells were collected, washed with cold PBS and suspended in binding buffer (0.1M Hepes ( $\mathrm{pH} 7.4$ ), 1.4 $\mathrm{M} \mathrm{NaCl}$ and $25 \mathrm{mM} \mathrm{CaCl}_{2}$ in solution; BD Biosciences). Following staining with $10 \mu \mathrm{l}$ PI, the cells were analyzed using a FACScan flow cytometer (BD Biosciences).

Cytokine content measurement in the tissue. The levels of IL-6, IL-1 $\beta$, and tumor necrosis factor (TNF)- $\alpha$ in the brains of the mice were measured in brain tissue using ELISA kits (R\&D Systems, Inc., Minneapolis, MN, USA), according to the manufacturer's instructions.

Reverse transcription-quantitative polymerase chain reaction ( $R T-q P C R)$. GL261 glioma cells were treated with IL-22 in vitro and cultured for $8 \mathrm{~h}$. IL-22 and IL-22 receptor (IL-22BP) mRNA expression levels in the brains of GL261 glioma-inoculated mice on days 0,7 and 14 were evaluated by RT-qPCR. Total RNA was extracted from the cells using an RNeasy mini kit (Qiagen China Co., Ltd., Beijing, China) according to the manufacturer's instructions. RT to cDNA was carried out using a Superscript III First Strand Synthesis kit (Invitrogen; Thermo Fisher Scientific, Inc.). qPCR was performed on an amplifier using real time PCR mix (Bio-Rad Laboratories, Inc., Hercules, CA, USA), RT products, $7.5 \mu 12 \mathrm{X}$ iQSYBR Green mix (Bio-Rad Laboratories, Inc.), $300 \mathrm{nM}$ forward and reverse primers and nanopure water to a final volume of $15 \mu \mathrm{l}$. Primer sequences were as follows: IL-22, forward AAGCATTGCCTTCTA GGTCTCC and reverse TCAGAGATACACGAGCTG GTT; IL-22BP, forward CATTGCCTTCTAGGTCTCCTCA and reverse CCTGCTTGCCAGTGCAAAAT; STAT3, forward CAATACCATTGACCTGCCGAT and reverse GAG CGACTCAAACTGCCCT; STAT4, forward GCAGCCAAC ATGCCTATCCA and reverse TGGCAGACACTTTGT GTTCCA; STAT6, forward CTCTGTGGGGCCTAATTT CCA and reverse CATCTGAACCGACCAGGAAC; Ki67, forward CGCAGGAAGACTCGCAGTTT and reverse CTG AATCTGCTAATGTCGCCAA; and GAPDH, forward AAT GGATTTGGACGCATTGGT and reverse TTTGCACTG GTACGTGTTGAT. PCR cycling conditions were as follows: $3 \mathrm{~min}$ at $95^{\circ} \mathrm{C}$ for the polymerase activation, 45 cycles of $10 \mathrm{sec}$ 
A

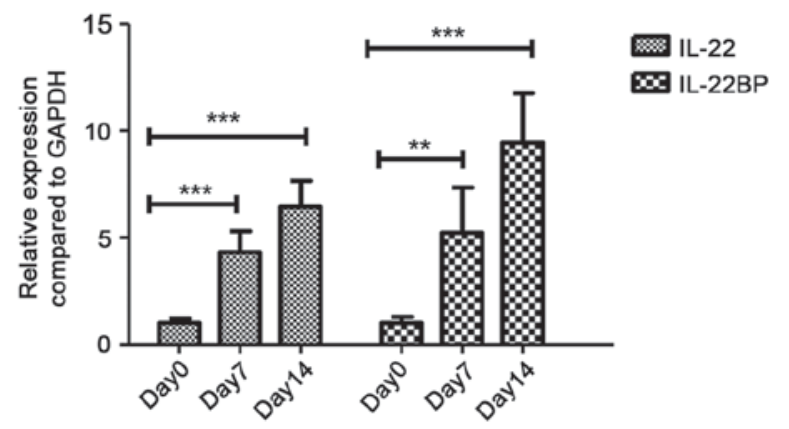

B

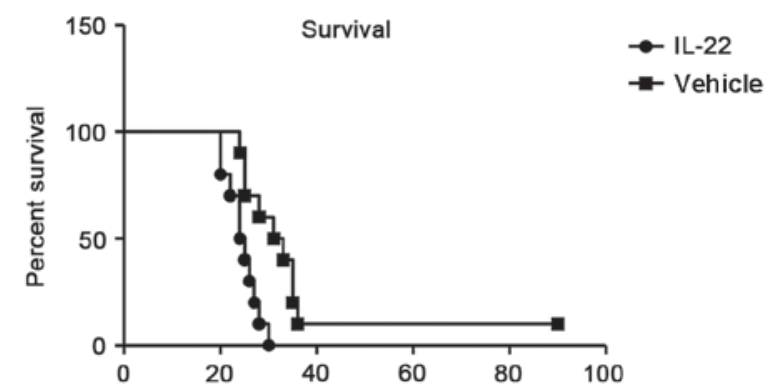

C

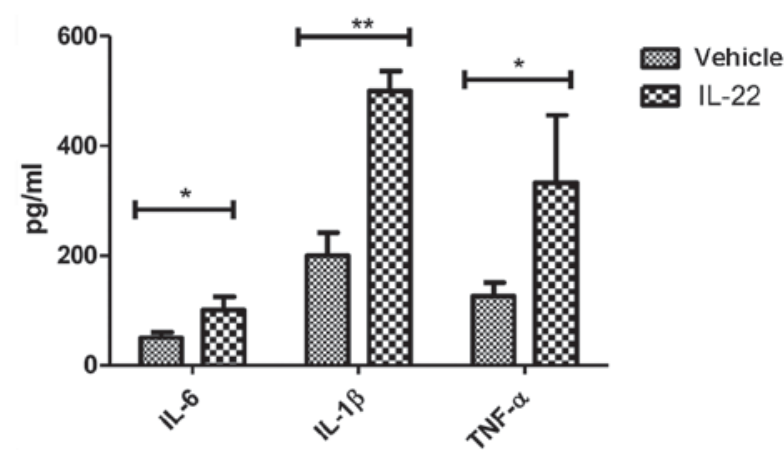

Figure 1. IL-22 injection promotes glioma development in vivo. (A) IL-22 and IL-22BP mRNA expression levels in the brains of GL261 glioma-inoculated mice on days 0,7 and 14 were detected by qPCR. (B) On day 5 after tumor implantation, $10 \mu \mathrm{l}$ of IL-22 (10 $\mu \mathrm{g} /$ mouse) or vehicle was injected through the same burr hole at the site of tumor inoculation. Mice were followed for the development of clinical symptoms. Data shown for the survival percentage are pooled from three independent experiments. (C) Effect of IL-22 on inflammatory IL- 6 , IL- $1 \beta$ and TNF- $\alpha$ levels was determined by ELISA. Student's $t$-test: ${ }^{*} \mathrm{P}<0.05,{ }^{* *} \mathrm{P}<0.01,{ }^{* * *} \mathrm{P}<0.001 ; \mathrm{n}=6$. Each experiment was performed in triplicate. IL, interleukin; qPCR, quantitative polymerase chain reaction; ELISA, enzyme-linked immunosorbent assay.

at $95^{\circ} \mathrm{C}$ for denaturation, and $30 \mathrm{sec}$ at $60^{\circ} \mathrm{C}$ for annealing and extension, followed by a DNA dissociation curve for the determination of the amplicon specificity. Analyses were performed in triplicate and water was used to replace DNA in samples used as negative controls. Data were analyzed using the $\mathrm{Cq}$ value normalized to the GAPDH endogenous reference gene. Data was collected and computed by iQ5 BioRad software with an automated analysis of the baseline and threshold of each run, and then exported in Excel files for further analyses.

Statistical analysis. The data are presented as the means \pm standard deviation. Statistical differences were determined using a two-tailed paired Student's t-test. SPSS software version 17.0 (SPSS, Inc., Chicago, IL, USA) was used to carry out the statis- tical analyses. $\mathrm{P}<0.05$ was considered to indicate a statistically significant result.

\section{Results}

IL-22 promotes glioma development in vivo. To determine the efficacy of IL-22 cytokine as a therapeutic treatment in the murine model of glioma, the expression levels of IL-22 were measured in the brain of GL261 glioma cell-inoculation mice at days 7 and 14. Significantly increased mRNA expression levels of IL-22 and IL-22BP were detected at day 7 and 14 compared with day 0 in the mouse glioma model (IL-22: day 7 vs. day $0, \mathrm{P}=0.001$; day 14 vs. day $0, \mathrm{P}<0.001$; and IL-22BP: day 7 vs. day $0, \mathrm{P}=0.009$; day 14 vs. day $0, \mathrm{P}<0.001$; Fig. $1 \mathrm{~A}$ ). To detect the biological function of IL-22 in the brain, IL-22 or vehicle were directly injected into the brain of normal mice to exclude the cytotoxic effect of IL-22 on the brain, the mice survived after local IL-22 treatment (data not shown). To assess the cytotoxic of IL-22 in vivo, IL-22 was injected into the healthy mice without glioma implantation, and the mice did not die after the single IL-22 injection. However, when the GL261 glioma cell was implanted into the cerebral hemisphere, the mice displayed severe disease following IL-22 injection, and significantly increased numbers of mice died compared with the vehicle-treated group ( $\mathrm{P}=0.008$; Fig. 1B). In addition, treatment with IL-22 significantly increased the expression levels of IL-6 $(\mathrm{P}=0.011), \mathrm{IL}-1 \beta(\mathrm{P}<0.001)$ and TNF- $\alpha(\mathrm{P}=0.018)$ in the brains of the mice, suggesting that IL-22 amplifies the immune response which is responsible for the brain tumor development in vivo (Fig. 1C)

IL-22 modulates cell proliferation through JAK-STAT-related gene expression. To elucidate the mechanism underlying the pathogenic role of IL-22 in glioma, the effect of IL-22 was examined in vitro. Firstly, the expression levels of the IL-22 receptor (IL-22BP) were determined. The expression of IL-22BP in the GL261 glioma cell line was detected, but IL-22 itself did not change the expression after IL-22 stimulation in vivo (Fig. 2A). The expression levels of the genes of the JAK/STAT signaling pathway were also investigated, and the cells were treated with $100 \mathrm{ng} / \mathrm{ml} \mathrm{IL-22} \mathrm{in} \mathrm{vitro.} \mathrm{IL-22}$ promoted the proliferation of glioma in vitro, which was indicated by the high expression levels of Ki67. As shown in Fig. 2B, IL-22 induced the expression of STAT3 $(\mathrm{P}=0.001)$ and STAT6 $(\mathrm{P}=0.006)$, but had no effect on STAT4 expression in GL261 cells, suggesting that IL-22 modulates neuronal inflammatory proteins through activation of STAT3/STAT6 in the JAK/STAT signaling pathway. Furthermore CCK8 staining demonstrated that IL-22 promoted cell proliferation (Fig. 2C), results which were supported by the increase in Ki67 expression observed using qPCR $(\mathrm{P}<0.001$; Fig. 2D). In addition, the cell death of the IL-22-treated cells was examined, and no significant difference was observed (Fig. 2E).

IL-22 KO attenuates glioma progression. To further evaluate the effect of IL-22 on glioma, the phenotype of IL-22 KO and IL-22 wild-type (WT) mice were compared in vivo. First, the expression levels of IL-22 in the glioma and brain of the recipient mice were investigated to confirm the efficacy of the IL-22 $\mathrm{KO}$, which indicated that IL-22 KO mice exhibited significantly 
A

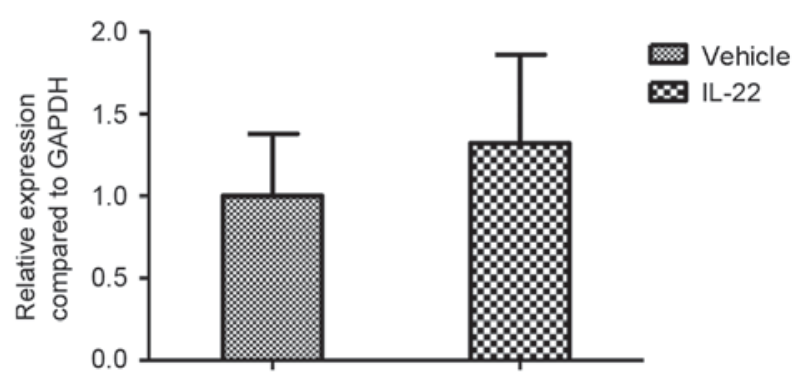

C

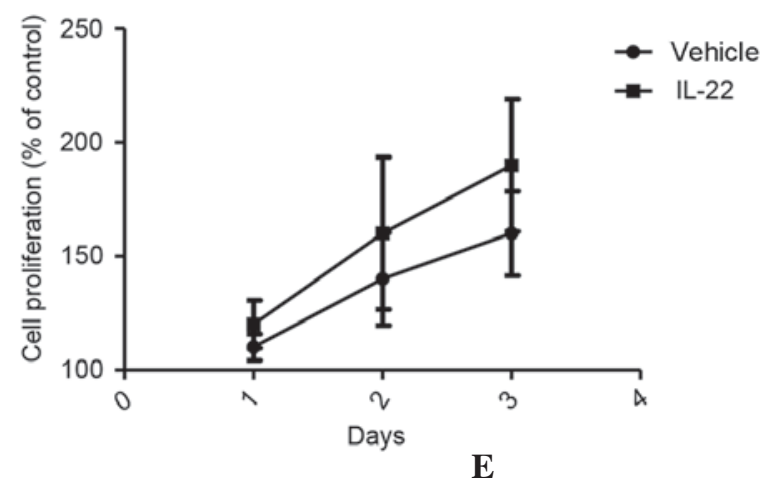

B
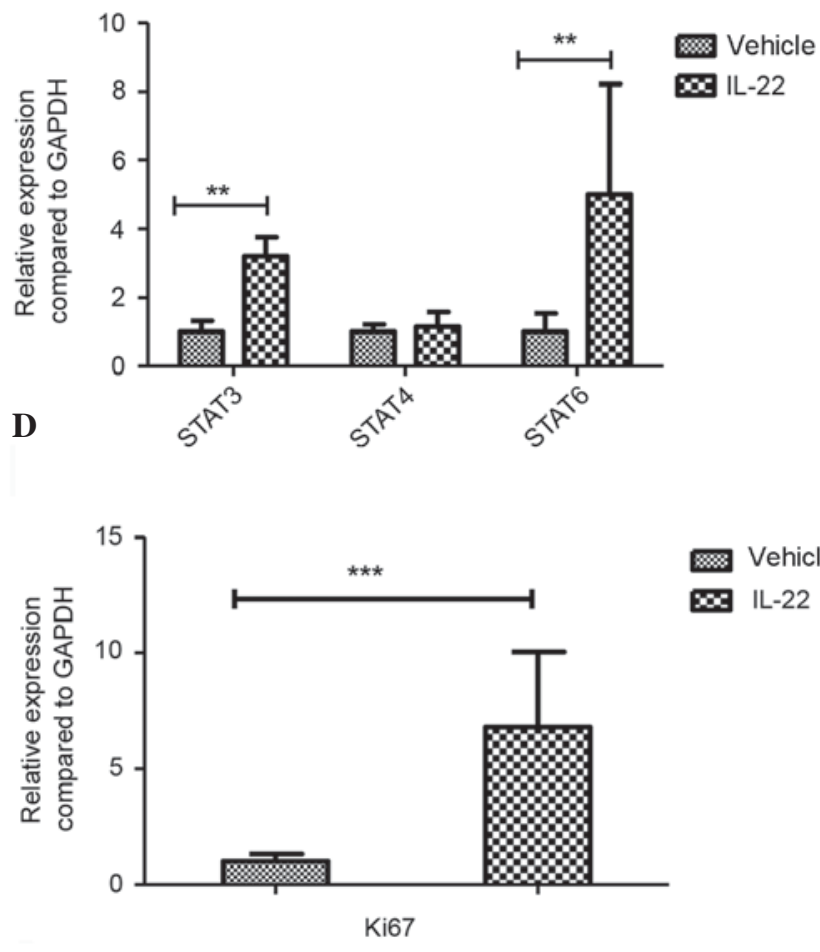

Vehicle

$\infty \mathrm{IL}-22$

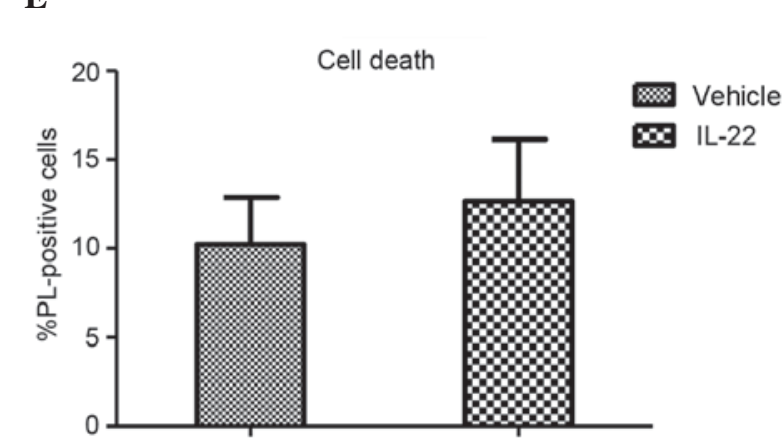

Figure 2. IL-22 treatment in vitro mediates the JAK/STAT signaling pathway and cell proliferation. (A) IL-22BP expression levels in the glioma following IL-22 treatment was detected by qPCR. (B) STAT family gene expression levels (STAT3, STAT4 and STAT6) following $100 \mathrm{ng} / \mathrm{ml}$ IL-22 treatment were detected by qPCR. (C) Cell proliferation (percentage of the control) after 3 days incubation with IL-22 or vehicle using CCK8 staining. (D) Ki67 gene expression levels were detected by qPCR on day 3. (E) The percentage of cell death measured as PI-positive cells (\%) was measured by flow cytometry. Student's $t$-test. ${ }^{* *} \mathrm{P}<0.01,{ }^{* * *} \mathrm{P}<0.001$. Each experiment was performed in triplicate. IL, interleukin; JAK, janus kinase; STAT, signal transducer and activator of transcription; qPCR, quantitative polymerase chain reaction; CCK8, cell counting kit 8.

diminished IL-12 expression in the brain $(\mathrm{P}<0.001$; Fig. 3A). The KO and WT mice were inoculated intracerebrally with GL261 glioma, and then the mice were observed for clinical symptoms. The survival of the IL-22 KO mice with glioma was significantly prolonged compared with the IL-22 WT mice (Fig. 3B). None of the surviving animals exhibited neurological disabilities. Tumor growth was observed to be the cause of death for all of the deceased animals. Furthermore, the inflammatory cytokines including IL-6 ( $\mathrm{P}=0.004), \mathrm{IL}-1 \beta(\mathrm{P}=0.001)$ and TNF- $\alpha(\mathrm{P}<0.001)$ were significantly reduced in the IL-22 KO mice (Fig. 3C), suggesting that the absence of IL-22, inflammatory cytokines which may be pathogenic for tumor development have decreased in the brain of glioma mice.

IL-22 blockade has a therapeutic effect on glioma. In order to develop a therapeutic approach for the treatment of IL-22,
anti-IL-22 mAb was utilized. Similar to IL-22 KO mice, the anti-IL-22 antibody alleviated the symptoms of mice glioma model, as identified by the higher survival percentage (Fig. 4A), and anti-IL-22 mAb reduced the levels of IL-6 $(\mathrm{P}<0.001)$, IL-1 $\beta(\mathrm{P}<0.001)$ and TNF- $\alpha(\mathrm{P}<0.001)$ inflammatory cytokines in the brain tissue, supporting the evidence for a role of anti-IL-22 mAb in glioma (Fig. 4B).

\section{Discussion}

In the US, $\sim 30,000$ new patients are diagnosed with glioma every year; glioblastoma is the most malignant form of glioma with a median survival of 14 months (18). Previous studies have demonstrated that gliomas retain many features of neuronal progenitor cells, including the ability to grow as neurospheres in culture, and the ability to self-renew and migrate in the 
A

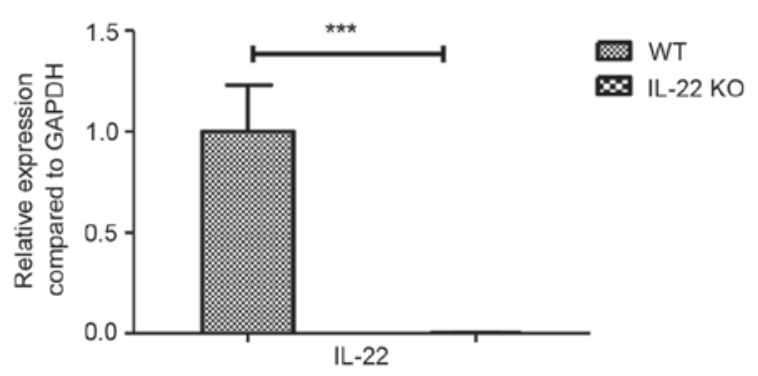

B

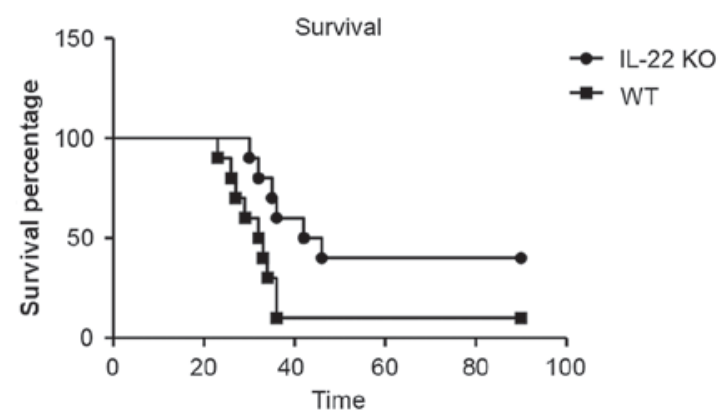

C

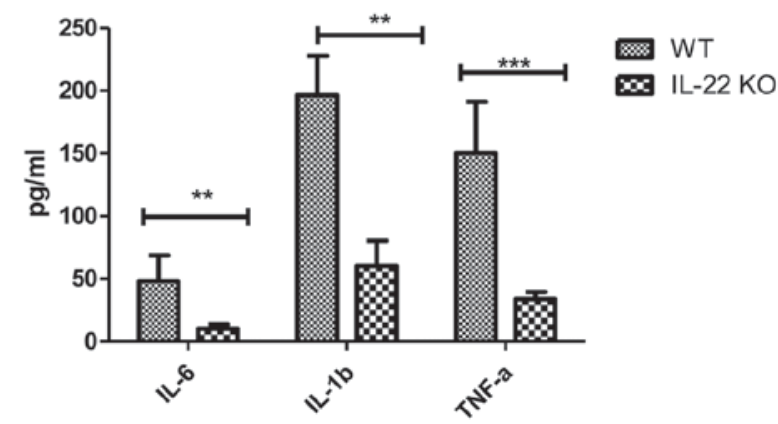

Figure 3. IL-22-deficient mice ameliorate glioma symptoms. (A) The IL-22 mRNA expression levels in the brain of IL-22 KO mice and WT mice were detected by qPCR. (B) IL-22 KO mice and WT mice were followed for the development of clinical symptoms following tumor implantation. Data shown for the survival percentage are pooled from three independent experiments. (C) Effect of IL-22 KO on inflammatory IL-6, IL-1 $\beta$ and TNF- $\alpha$ levels was determined by ELISA. Student's $t$-test: ${ }^{*} \mathrm{P}<0.05,{ }^{* *} \mathrm{P}<0.01,{ }^{* * *} \mathrm{P}<0.001 . \mathrm{n}=6$. Each experiment was performed in triplicate. IL, interleukin; qPCR, quantitative polymerase chain reaction; ELISA, enzyme-linked immunosorbent assay; WT, wild-type; KO, knock-out; TNF, tumor necrosis factor.

brain $(19,20)$. However, inflammation-related cytokines and chemokines may have important roles in different types of cancer. It has been reported that miR-124 inhibits STAT3 signaling to enhance $\mathrm{T}$ cell-mediated immune clearance of glioma by recruiting IL- 2 , IFN- $\gamma$ and TNF- $\alpha$ cytokines $(21,22)$. The results of the present study demonstrated that IL-22 promotes tumor growth in the brain by regulating inflammatory cytokine production and cell proliferation, suggesting that IL-22 may be a candidate for the therapeutic targeting of glioma.

Higher expression levels of IL-22 and IL-22 receptor (IL-22BP) were observed in the mouse glioma model. IL-22 injection reduced the survival percentage of glioma-inoculated mice, suggesting a role for IL-22 in tumor growth. To further confirm the effect of IL-22 on glioma, inflammatory cytokine levels were measured using ELISA. It was revealed that IL-6,

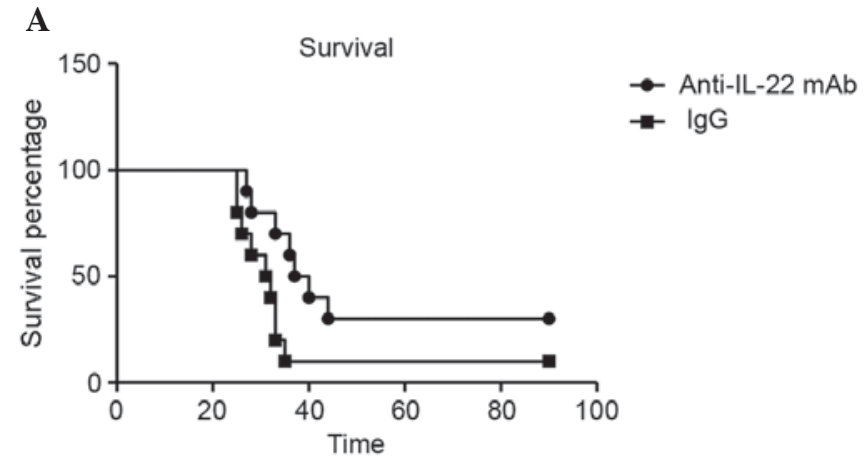

B

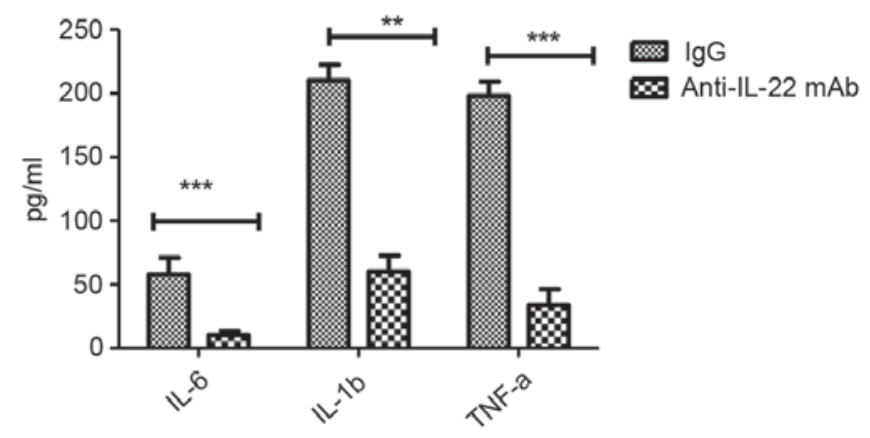

Figure 4. Anti-IL-22 antibody ameliorates glioma severity. (A) On day 5 after tumor implantation, $10 \mu \mathrm{l}$ of IL-22 mAb (100 $\mu \mathrm{g} /$ mouse) or control IgG was injected through the same burr hole at the site of tumor inoculation. Mice were followed for the development of clinical symptoms. Data shown for the survival percentage are pooled from three independent experiments. (B) Effect of anti-IL-22 mAb on inflammatory IL-6, IL-1 $\beta$ and TNF- $\alpha$ levels was determined by ELISA. Student's $t$-test: ${ }^{* *} \mathrm{P}<0.01,{ }^{* * *} \mathrm{P}<0.001 . \mathrm{n}=6$. Each experiment was performed in triplicate. IL, interleukin; qPCR, quantitative polymerase chain reaction; ELISA, enzyme-linked immunosorbent assay; TNF, tumor necrosis factor; IgG, immunoglobulin G; mAB, monoclonal antibody.

IL-1 $\beta$, and TNF- $\alpha$ were induced in vivo by IL-22 injection. Concordant with these findings, the expression of IL-22BP were detected in the glioma cell line, and cells were treated with IL-22 in vitro to elucidate the mechanism underlying IL-22 function in vivo. It was demonstrated that IL-22 was able to increase the mRNA expression levels of STAT3 and STAT6, thus activating the JAK/STAT signaling pathway. In fact, various cytokines mediated STAT activation, including IL-4 that was observed to specifically activate STAT6, and IL-12 that modulated STAT4 (23-25). In addition, IL-22 induced the phosphorylation of STAT3 $(17,26)$. Previous studies reported that JAK1 and STAT3 expression levels were higher in low grade gliomas, as compared with high grade gliomas, although the factors that induce STAT3 gene expression remain to be determined $(27,28)$. Consequently, the results of the present study demonstrated that IL-22 promoted the proliferation of glioma in vitro, which was indicated by the high expression levels of Ki67. These results suggest that STAT3 and STAT6 are involved in the process of IL-22-mediated proliferation and regulation of the JAK/STAT signaling pathway in vitro, could result in the phenotype in vivo.

In addition, the effects of IL-22 on glioma were further confirmed using the IL-22 KO mice. Mice with glioma deficient in IL-22 showed alleviated disease severity. Consistently, decreased levels of inflammatory cytokines were observed in the IL-22 KO mice. It was previously reported that glioma 
cells produce cytokines with an anti-inflammatory phenotype, including IL-10, IL-4, IL-6, TGF- $\beta$, and prostaglandin E2 (29). TGF- $\beta$ in particular suppresses the activation and proliferation of microglia (30); the results of the present study demonstrated that IL-6, IL-1 and TNF- $\alpha$ expression was downregulated in the IL-22 KO mouse glioma model, thus IL-22 may globally regulate different cytokines, including IL-6 and IL-1. Indeed, IL-22 secreted by Th17 cells may elicit the production of IFN- $\gamma$, which is also different from the cytokines in the CNS. Similarly, using IL-22-blocking antibodies was demonstrated to protect mice from glioma growth with a higher survival percentage, whilst preventing the secretion of inflammatory cytokines and an immune response in the brain. Both the IL-22 KO and anti-IL-22 antibody protected the mice from glioma. To our knowledge, the present study is the first to demonstrate IL-22 efficacy in a mice glioma model in vivo and in vitro. The results demonstrated that IL-22 has an important role in glioma via the regulation of neuronal apoptosis, inflammatory cytokines, neural proliferation, and JAK/STAT signaling in the tumor microenvironment of the CNS. Anti-IL-22 antibody may prove useful in the treatment of gliomas in clinical settings.

\section{Acknowledgements}

The present study was supported by a grant from the Natural Science Foundation of Hubei Province, China (grant no. 050612036).

\section{References}

1. Yamanaka R, Abe T, Yajima N, Tsuchiya N, Homma J, Kobayashi T, Narita M, Takahashi M and Tanaka R: Vaccination of recurrent glioma patients with tumour lysate-pulsed dendritic cells elicits immune responses: Results of a clinical phase I/II trial. Br J Cancer 89: 1172-1179, 2003.

2. Yu JS, Liu G, Ying H, Yong WH, Black KL and Wheeler CJ: Vaccination with tumor lysate-pulsed dendritic cells elicits antigen-specific, cytotoxic T-cells in patients with malignant glioma. Cancer Res 64: 4973-4979, 2004.

3. Liau LM, Prins RM, Kiertscher SM, Odesa SK, Kremen TJ, Giovannone AJ, Lin JW, Chute DJ, Mischel PS, Cloughesy TF and Roth MD: Dendritic cell vaccination in glioblastoma patients induces systemic and intracranial T-cell responses modulated by the local central nervous system tumor microenvironment. Clin Cancer Res 11: 5515-5525, 2005.

4. Valle RD, de Cerio AL, Inoges S, Tejada S, Pastor F, Villanueva $\mathrm{H}$, Gallego J, Espinos J, Aristu J, Idoate MA, et al: Dendritic cell vaccination in glioblastoma after fluorescence-guided resection. World J Clin Oncol 3: 142-149, 2012.

5. Fabry Z, Raine CS and Hart MN: Nervous tissue as an immune compartment: The dialect of the immune response in the CNS. Immunol Today 15: 218-224, 1994

6. Carmen J, Gowing G, Julien JP and Kerr D: Altered immune response to CNS viral infection in mice with a conditional knock-down of macrophage-lineage cells. Glia 54: 71-80, 2006.

7. Andersson U and Tracey KJ: Neural reflexes in inflammation and immunity. J Exp Med 209: 1057-1068, 2012.

8. Palucka $\mathrm{K}$ and Banchereau J: Cancer immunotherapy via dendritic cells. Nat Rev Cancer 12: 265-277, 2012.

9. Almolda B, Costa M, Montoya M, Gonzàlez B and Castellano B: Increase in Th17 and T-reg lymphocytes and decrease of IL22 correlate with the recovery phase of acute EAE in rat. PloS One 6: e27473, 2011.

10. Cheng F, Guo Z, Xu H, Yan D and Li Q: Decreased plasma IL22 levels, but not increased IL17 and IL23 levels, correlate with disease activity in patients with systemic lupus erythematosus. Ann Rheum Dis 68: 604-606, 2009.
11. Van Belle AB, de Heusch M, Lemaire MM, Hendrickx E, Warnier G, Dunussi-Joannopoulos K, Fouser LA, Renauld JC and Dumoutier L: IL-22 is required for imiquimod-induced psoriasiform skin inflammation in mice. J Immunol 188: 462-469, 2012.

12. Jiang R, Tan Z, Deng L, Chen Y, Xia Y, Gao Y, Wang X and Sun B: Interleukin-22 promotes human hepatocellular carcinoma by activation of STAT3. Hepatology 54: 900-909, 2011.

13. Lejeune D, Dumoutier L, Constantinescu S, Kruijer W, Schuringa JJ and Renauld JC: Interleukin-22 (IL-22) activates the JAK/STAT, ERK, JNK and p38 MAP kinase pathways in a rat hepatoma cell line. Pathways that are shared with and distinct from IL-10. J Biol Chem 277: 33676-33682, 2002.

14. Zhang W, Chen Y, Wei H, Zheng C, Sun R, Zhang J and Tian Z: Antiapoptotic activity of autocrine interleukin-22 and therapeutic effects of interleukin-22-small interfering RNA on human lung cancer xenografts. Clin Cancer Res 14: 6432-6439, 2008.

15. Sabat R, Ouyang W and Wolk K: Therapeutic opportunities of the IL-22-IL-22R1 system. Nat Rev Drug Discov 13: 21-38, 2014.

16. Kreymborg K, Etzensperger R, Dumoutier L, Haak S, Rebollo A, Buch T, Heppner FL, Renauld JC and Becher B: IL-22 is expressed by Th17 cells in an IL-23-dependent fashion, but not required for the development of autoimmune encephalomyelitis. J Immunol 179: 8098-8104, 2007.

17. Backert I, Koralov SB, Wirtz S, Kitowski V, Billmeier U, Martini E, Hofmann K, Hildner K, Wittkopf N, Brecht K, et al: STAT3 activation in Th17 and Th22 cells controls IL-22-mediated epithelial host defense during infectious colitis. J Immunol 193: 3779-3791, 2014.

18. Davis FG and McCarthy BJ: Current epidemiological trends and surveillance issues in brain tumors. Expert Rev Anticancer Ther 1: 395-401, 2001.

19. Colleoni F and Torrente Y: The new challenge of stem cell: Brain tumour therapy. Cancer Lett 272: 1-11, 2008.

20. Singh SK, Hawkins C, Clarke ID, Squire JA, Bayani J, Hide T, Henkelman RM, Cusimano MD and Dirks PB: Identification of human brain tumour initiating cells. Nature 432: 396-401, 2004.

21. Silber J, Lim DA, Petritsch C, Persson AI, Maunakea AK, Yu M, Vandenberg SR, Ginzinger DG, James CD, Costello JF, et al: miR-124 and miR-137 inhibit proliferation of glioblastoma multiforme cells and induce differentiation of brain tumor stem cells. BMC Med 6: 14, 2008.

22. Wei J, Wang F, Kong LY, Xu S, Doucette T, Ferguson SD, Yang Y, McEnery K, Jethwa K, Gjyshi O, et al: MiR-124 inhibits STAT3 signaling to enhance T cell-mediated immune clearance of glioma. Cancer Res 73: 3913-3926, 2013.

23. Kaplan MH, Schindler U, Smiley ST and Grusby MJ: Stat6 is required for mediating responses to IL-4 and for development of Th2 cells. Immunity 4: 313-319, 1996.

24. Monteleone G, Holloway J, Salvati VM, Pender SL, Fairclough PD, Croft N and MacDonald TT: Activated STAT4 and a functional role for IL-12 in human Peyer's patches. J Immunol 170: 300-307, 2003.

25. Morinobu A, Gadina M, Strober W, Visconti R, Fornace A, Montagna C, Feldman GM, Nishikomori R and O'Shea JJ: STAT4 serine phosphorylation is critical for IL-12-induced IFN-gamma production but not for cell proliferation. Proc Natl Acad Sci USA 99: 12281-12286, 2002.

26. Sovran B, Loonen LM, Lu P, Hugenholtz F, Belzer C, Stolte EH, Boekschoten MV, van Baarlen P, Kleerebezem M, de Vos P, et al: IL-22-STAT3 pathway plays a key role in the maintenance of ileal homeostasis in mice lacking secreted mucus barrier. Inflamm Bowel Dis 21: 531-542, 2015.

27. Zhang L, Liu W, Alizadeh D, Zhao D, Farrukh O, Lin J, Badie SA and Badie B: S100B attenuates microglia activation in gliomas: Possible role of STAT3 pathway. Glia 59: 486-498, 2011.

28. Lo HW, Cao X, Zhu H and Ali-Osman F: Constitutively activated STAT3 frequently coexpresses with epidermal growth factor receptor in high-grade gliomas and targeting STAT3 sensitizes them to Iressa and alkylators. Clin Cancer Res 14: 6042-6054, 2008.

29. Zisakis A, Piperi C, Themistocleous MS, Korkolopoulou P, Boviatsis EI, Sakas DE, Patsouris E, Lea RW and Kalofoutis A: Comparative analysis of peripheral and localised cytokine secretion in glioblastoma patients. Cytokine 39: 99-105, 2007.

30. Suzumura A, Sawada M, Yamamoto H and Marunouchi T: Transforming growth factor-beta suppresses activation and proliferation of microglia in vitro. J Immunol 151: 2150-2158, 1993. 